\title{
DUPLICATION OF THE PITUITARY AND STOMATODAEAL STRUCTURES IN A 38-WEEK MALE INFANT
}

\author{
BY \\ W. R. M. MORTON \\ From the Department of Anatomy, Queen's University, Belfast
}

(RECEIVED FOR PUBLICATION OCTOBER 8, 1956)

Duplication of the pituitary (hypophysis) and other structures in the region of the mouth may be regarded as a minor degree of partial twinning which did not go on to frank monster formation. Ahlfeld (1880), who considered that the first grade of duplication of the anterior end of the body was a doubling of the hypophysis, reports one case in man; and McCall (1943) records a double Rathke's pouch with two corresponding diencephalic diverticula in a $10-\mathrm{mm}$. C.R. rat embryo. No other reports of such duplications have been found in a search of the literature. These abnormalities are interesting not only because of their rarity, but also because they illustrate that twin formation may well occur, as postulated by Corner (1955), during the differentiation of the germ disc from the inner cell mass.

\section{History}

A 38-week male infant, born on March 11, 1956, of a primigravida aged 28 years, was sent to hospital on account of a double cleft palate and other malformations of the interior of the mouth. He died three days after birth and a full anatomical examination of the body was carried out after perfusion through the femoral arteries.

There was no history of congenital malformations in either the mother's or the father's family. The mother gave no history of any illness from early childhood, and had no pyrexial upsets during the pregnancy. Her menstrual periods were 'normal and regular'. Her last menstrual period had been on June 16, 1955. Slight uterine bleeding lasting 36 hours was noted on June 30 , and a continuous loss lasting 21 days started on July 14, 1955.

\section{General Description}

The external appearance of the child was normal except that the mouth was wide, the lower lip was duplicated and the chin showed a central supernumerary prominence (Fig. 1). Inside the mouth two frenula, one on either side of the mid-line, connected the upper lip to the gum (Fig. 2). Two supernumerary central incisor teeth were contained in the gum between the frenula. Two mandibles, each with a distinct symphysis, were present (Fig. 3). The medial parts of these bones ended in a firm bar of tissue connecting the floor to the roof of the mouth. It was covered by squamous epithelium from which numerous racemose glands arose, and in the upper part consisted of large masses of striped muscle and some blood vessels. The vessels ran upwards through a gap in the sphenoid to the interior of the skull. A tongue lay within each mandibular arch, the left being the broader and more horizontal of the two. Posteriorly they were united to form a single broad lingual mass on which a single row of circumvallate papillae was present (Fig. 4). The muscles of the tongues and lower jaws, attached to the broad hyoid bone, could not be readily identified, but the right hypoglossal nerve supplied musculature in both tongues, while the left only supplied that on the left side.

A complete bilateral cleft palate was present, the clefts running backwards from behind the alveolus, and the oral and nasal cavities communicated freely with each other. A soft, whitish mass covered with fine hairs hung down from the nasal septum posterior to the alveolar arch. A second polypoid mass, almost completely obstructing the nasopharynx, hung down from the roof of the mouth behind and to the left of the upper attachment of the abnormal bar of tissue; it separated the uvular ends of the right and left palatal processes (Fig. 2). A tuft of long black hairs protruded downwards between this second mass and the abnormal bar. The upper border of the epiglottis was slightly notched, but no other evidence of duplication was found in the larynx, trachea, oesophagus, thoracic or abdominal viscera, or in the limbs. 


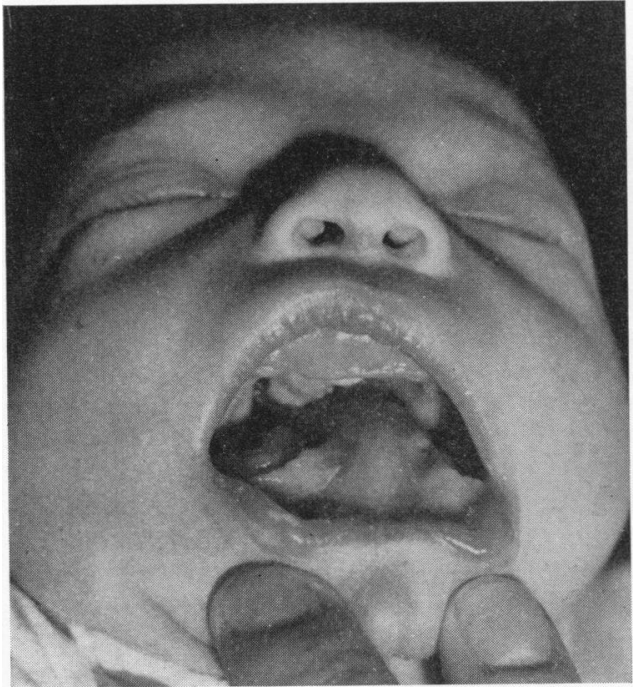

FIG. 1.

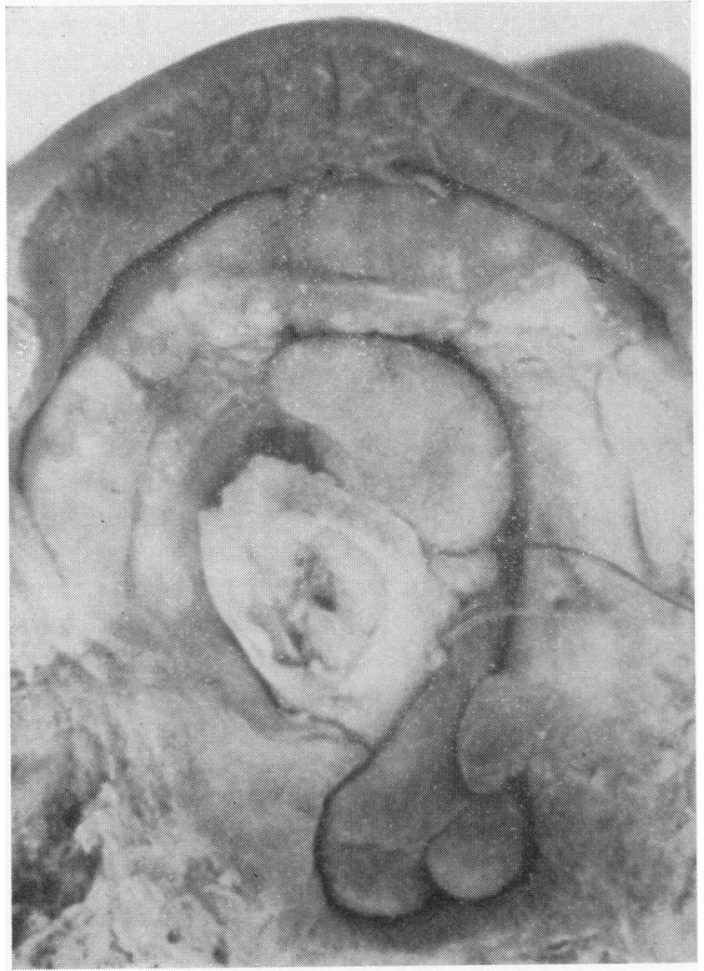

FIG. 2.

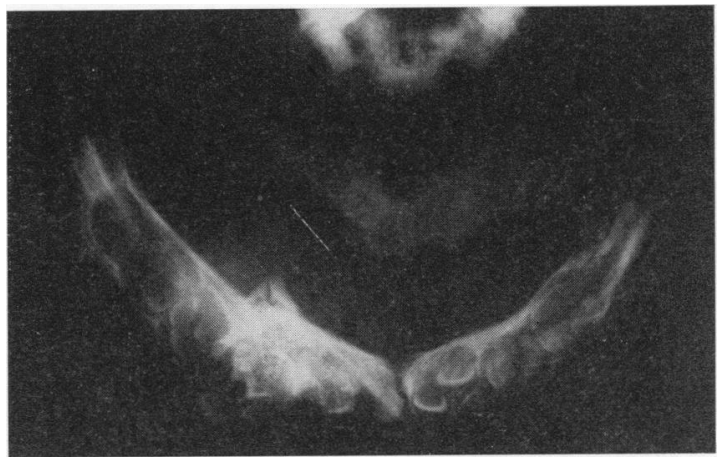

FIG. 3.

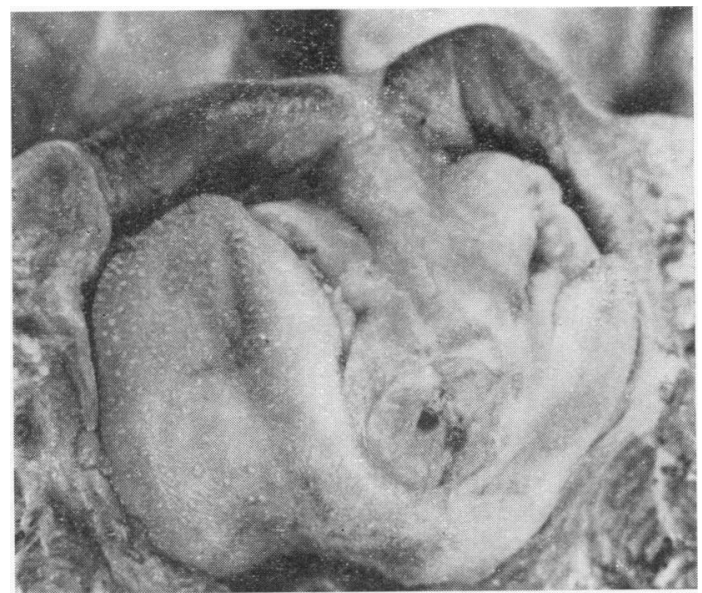

FIG. 4.

FIG. 1.-View of the mouth showing the right tongue, the abnorma bar with the alveolar margins, the double lip and the midline chir prominence.

Fig. 2.-The roof of the mouth. Two frenula join the upper lip tc the gum, behind which lies a hair-covered polyp. Long hairs lie tc the left of the cut end of the abnormal bar, and a polyp, covered with squamous epithelium, separates the uvular ends of the palate posteriorly. $\times 1 \cdot 1$.

FIG. 3.-Radiograph of the lower jaw. A well-marked symphysis separates the left half mandible from the medial mass of bone. A second symphysis can also be seen to the right of the midline.

Fig. 4-The floor of the mouth. The double lower lips and lowes alveolar ridges can be seen anterior to the two tongues. The cul lower end of the abnormal bar lies immediately anterior to the fusec posterior lingual mass. $\times 1 \cdot 6$. 


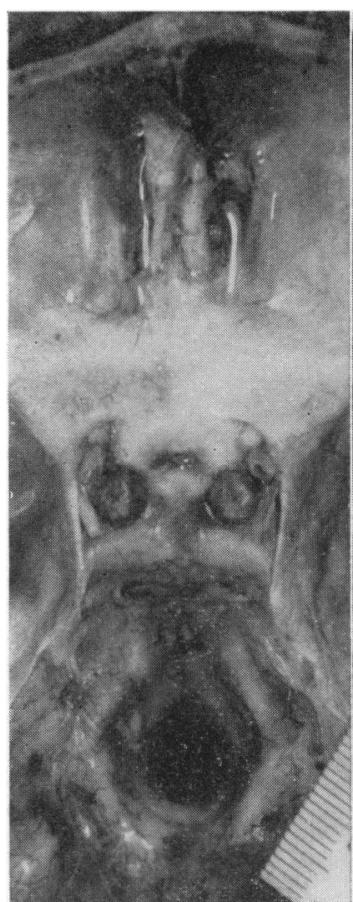

FIG. 5.

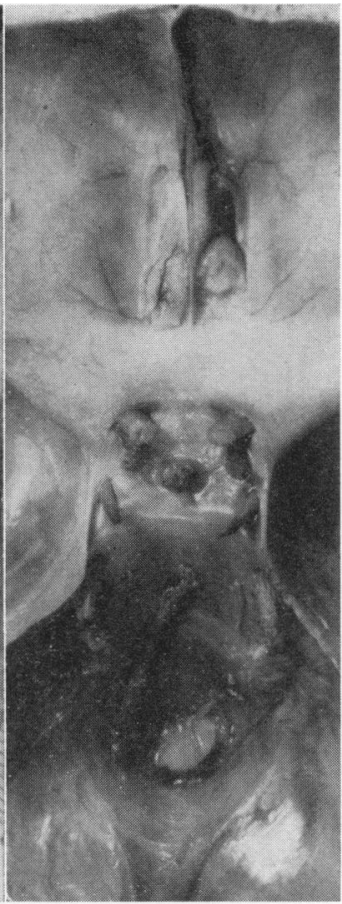

FiG. 6.

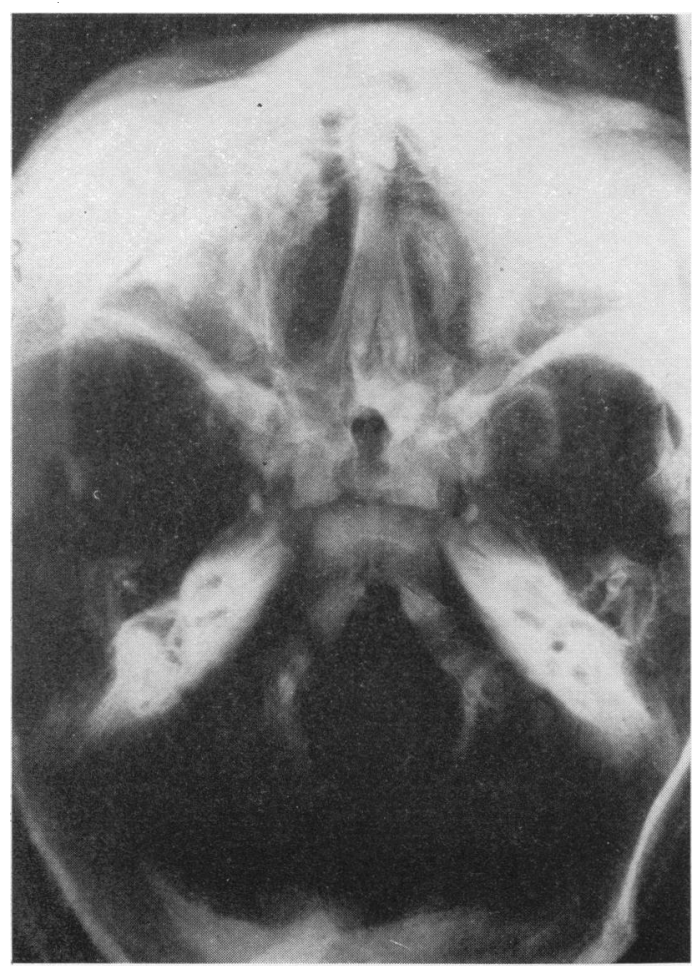

FIG. 7.

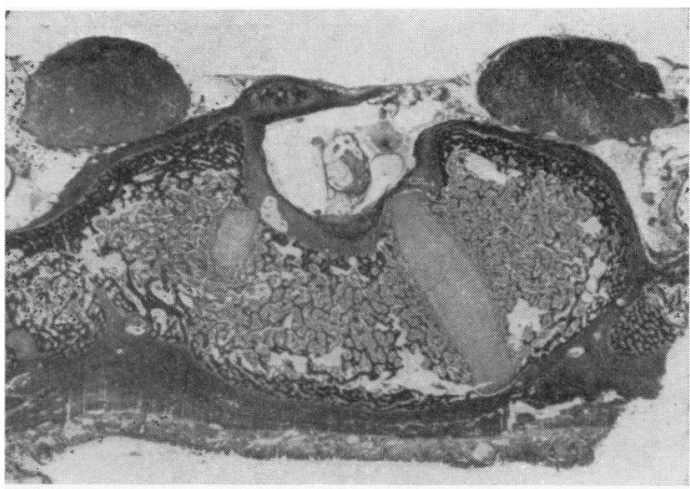

FiG. 8.

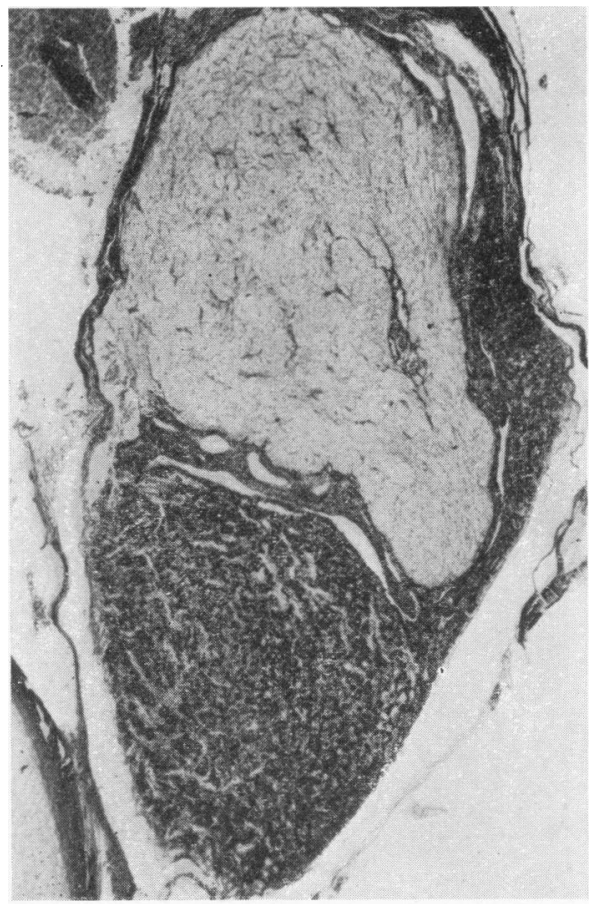

FIG. 9.

Fig. 5.-Base of the skull from above. The two hypophyses lie behind the optic nerves and internal carotid arteries. The depression antero-medial to the glands is referred to in Fig. 7 . $\times 0.9$.

FIG. 6.-The base of the skull in a normal full-term male infant to show the relations of the hypophysis. $\times 0.9$.

FIG. 7.-Radiograph of the base of the skull after removal of the brain. The midline bony defect in the sellar region corresponds to the depression between the two hypophyses and transmitted blood vessels from the upper end of the abnormal bar to the interior of the skull.

FIG. 8.-Coronal section through the sellar region. The pars anterior of each pituitary lies laterally and blood vessels lie in a midline vacuity. Bars of cartilage subdivide the bone in an unusual manner. Ciliated columnar epithelium lines the pharynx in this region. $\times 3 \cdot 7$.

FIG. 9.-Low-power view of the left hypophysis showing the neural lobe, pars intermedia, cleft and the pars anterior. $\times 30$. 


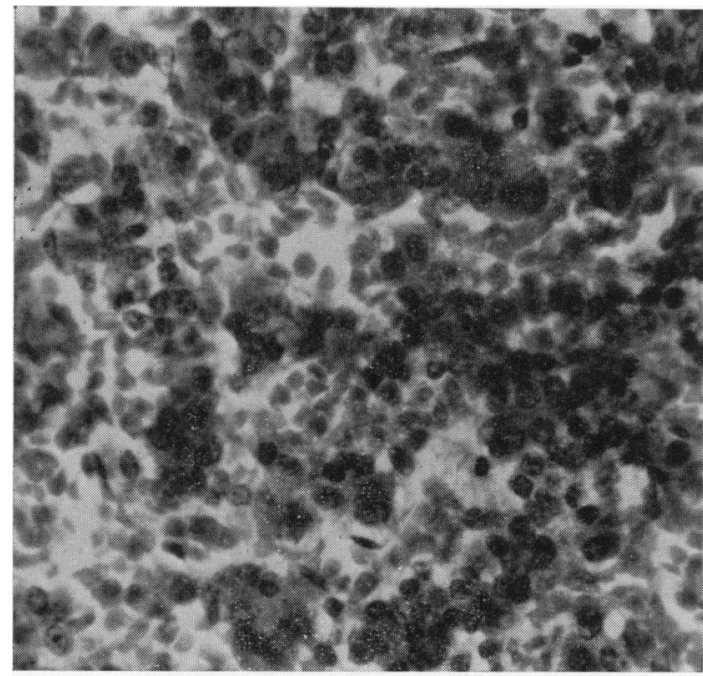

FIG. 10.

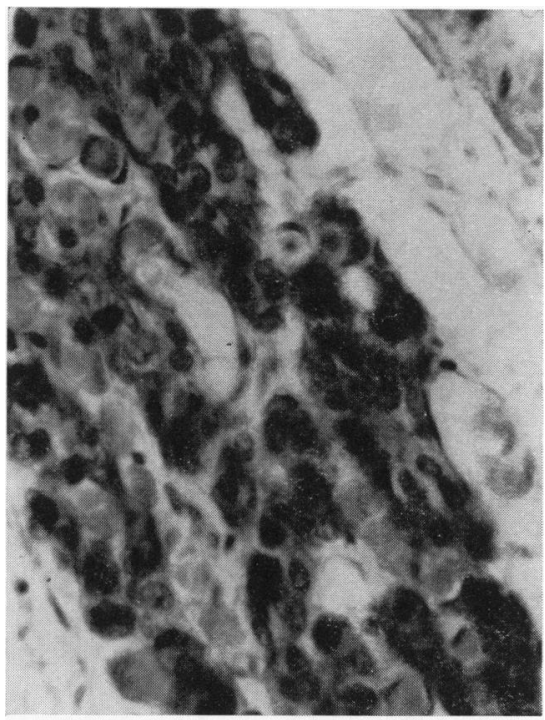

FIG. 11.

FIG. 10.-High-power view of the pars anterior. Blood-filled capillaries show as light areas. $\times \mathbf{4 4 0}$.

FIG. 11.-High-power view of a portion of the right pharyngeal hypophysis. The cells are not so tightly packed as in the main glands. $\times 440$.

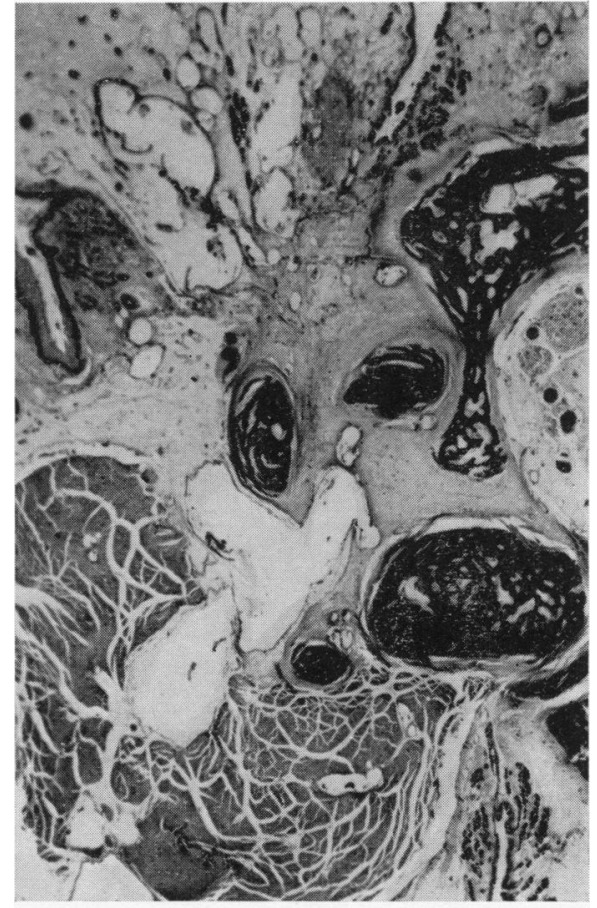

FIG. 12.

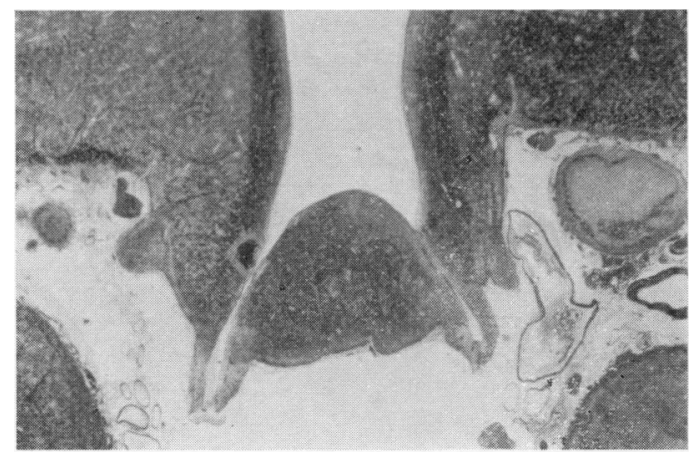

FIG. 13.

FIG. 12.-The upper attachment of the abnormal bar of tissue. Striped muscle covered by a squamous epithelium forms the bulk of this bar to the left of which is a mucoid polyp. The numerous mucous glands lie beside the adeno-hypophyseal tissue which is attached to the columnar epithelium in the depths of the laterallyplaced crypts. $\quad \times 7 \cdot 25$.

FIG. 13.-Coronal section of the brain. The two hollow hypophyseal stalks are separated by an elevation in the floor of the third ventricle, which contains numerous nerve cells. $\times 3.7$. 
Base of the Skull. The outstanding feature of the interior of the skull was the presence of two pituitary glands lying on either side of the midline posteromedial to the upturned ends of the internal carotid arteries (Figs. 5 and 6). Antero-medial to the glands there was a slight depression which corresponded in position to the absence of bone as seen on a radiograph (Fig. 7). Each hypophysis measured approximately $5 \mathrm{~mm}$. in both antero-posterior and transverse diameters, and from 4 to $4.5 \mathrm{~mm}$. vertically. They were more ovoid in coronal section and without the broad base seen in the hypophysis of a normal full-term male infant, which measured 6,7 and $5 \mathrm{~mm}$. in comparable diameters. The optic nerves were $17 \mathrm{~mm}$. apart at the optic foramina as compared with $10 \mathrm{~mm}$. in the normal, and the chiasma was correspondingly broadened.

Serial sections cut coronally at 10 microns were made of the base of the skull so as to include the whole of the hypophyses and the upper part of the naso-pharynx (Fig. 8). All the hypophyseal sections and every twelfth section anterior to that were mounted. Sections were stained with haematoxylin and eosin, pyrrol and eosin, Weigert and van Gieson stains, Mallory's or Masson's triple stains.

Histologically each hypophysis (Fig. 9) consisted of a neuro- and an adeno-hypophysis. The neural lobes, pars intermedia and clefts were small but otherwise normal. The adeno-hypophyses showed all the characters of normal glands both in vascularity, general arrangement of the cells and in their staining reactions (Fig. 10). Large accessory adenohypophyseal masses, presumably formed from the stems of Rathke's pouches, were found in the pharyngeal roof. These masses measured $1.4 \mathrm{~mm}$. by $0.6 \mathrm{~mm}$. on an average, and extended through 675 sections on the right side and 820 sections on the left side. They were composed of cells identical in appearance and staining reactions with those of the main adeno-hypophysis, and were equally vascular (Fig. 11). The mass on the left side was attached by a short diverticulum, and that on the right by a solid cord of cells to the epithelium of the pharynx. This epithelium was columnar at the points of attachment but became squamous and contained numerous hair follicles a short distance medial to these points (Fig. 12).

The Brain. The olfactory tracts were not present. Two hollow hypophyseal stalks projected downwards from the floor of the third ventricle behind the wide chiasma and anterior to two mammillary bodies. No other abnormalities were found in the brain. Coronal sections, at 10 microns, of the hypophyseal stalks and lateral walls of the third ventricle were made and stained for nerve fibres (Foot's modification of the Bielschowsky technique) and for cells (gentian violet stain). The floor of the third ventricle showed a median elevation separating two depressions which led down to the stalks (Fig. 13). Nerve cells were present in this elevation but no definite arrangement into nuclei could be made out. Normal supra-optic nuclei were present laterally. For comparison, the brain and hypophyseal region of a normal infant were also examined.

\section{Discussion}

Simple failure of union of embryonic masses around the stomatodaeum, such as led in Bosatra's (1955) case to a bifid tongue and median cleft in the upper lip, could not have caused the duplications described above. It is necessary, in order to explain them, to envisage a stage in early development during which a small deviation from the normal could occur which would influence the hypophyseal and oral regions and, by reason of the secondary chain of events initiated by it, bring about considerable changes in the head region. Duplication of cells of the prochordal plate and of the anterior end of the notochordal process at the fifteenth to sixteenth day of pregnancy would produce such changes, as it is these tissues which act on the overlying ectoderm inducing differentiation of the anterior end of the germ disc. Why duplication should have occurred, and the means whereby it was caused, are unknown, but, as Töndury (1955) states in his review of the problem of the developmental physiology of abnormal growth, physical and chemical factors must act at critical phases to produce abnormalities. Maternal ill health due to rubella is known to produce neural lesions, and avitaminosis (Ancel, 1954), hypervitaminosis (Giroud and Martinet, 1955) and other agents have been used experimentally in the production of foetal abnormalities (Fraser and Fainstat, 1951). No history of maternal ill health during the critical period of the pregnancy was discovered in this case, but the uterine bleeding, starting at about the fourteenth day of gestation and lasting 21 days, may well have a causal relationship.

A brief description of the normal development of the pituitary, based on the work of Gilbert (1935), will help in following the probable course of events leading to the production of the abnormalities.

The hypophysis develops, in the rat, from a proliferation of the undifferentiated ectoderm common to the neural plate and stomatodaeum, at the anterior end of the medullary groove. This ectoderm is situated just anterior to the stomatodaeal 
(a)

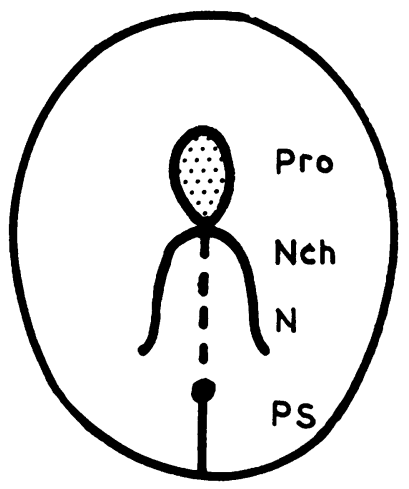

(c)

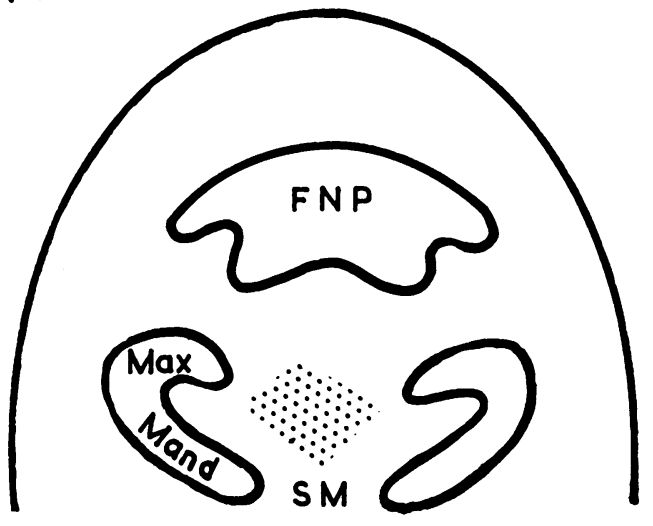

(b)

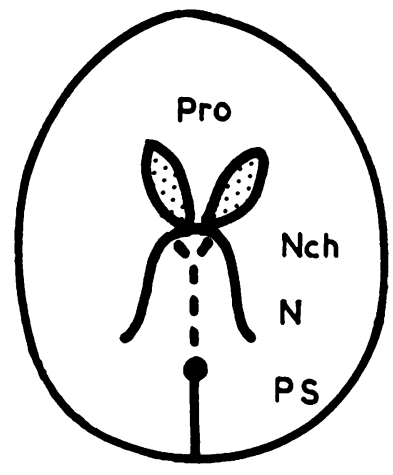

(d)

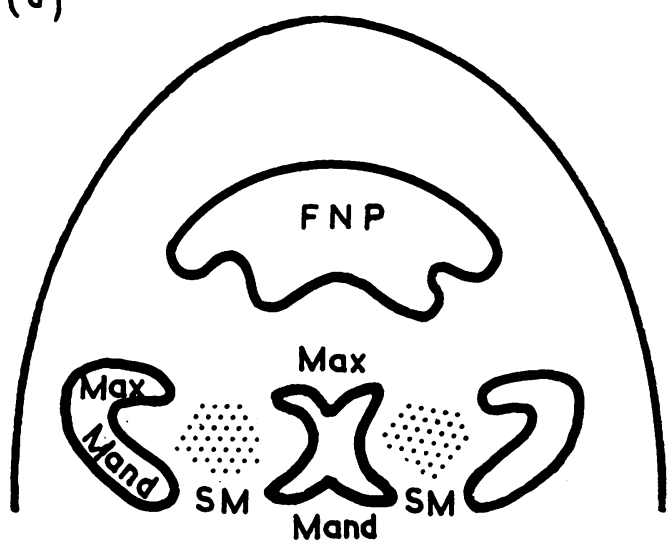

Fig. 14.-Diagram to illustrate the early development of a normal embryo as contrasted with what may have occurred in the present case. The embryonic shield is shown as if transparent and viewed from above in $(a)$ and (b), and the stomatodaeal region as from in front and below in $(c)$ and $(d)$.

(a) The normal embryonic shield.

(b) Duplication of prochordal plate and anterior end of notochordal process.

(c) Normal mesoderm masses around the stomatodaeum.

(d) Duplication of mandibular and maxillary mesoderm between two stomatodaeal membranes.

(or oral) membrane and the notochord. The definitive neural and surface ectoderms form from this mass, and mesoderm then separates the neural tube from the surface ectoderm, except at the region of the developing hypophysis, where the initial apposition of ectoderms persists. Increase in the amount of this mesoderm produces the diverticulum known as Rathke's pouch. Specialized masses of mesoderm, from which the nose and upper and lower jaws are formed, develop into the fronto-nasal

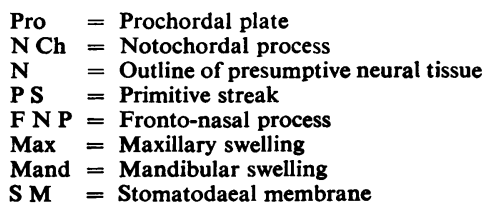

process above, and the mandibular and maxillary swellings on either side of the stomatodaeum.

In the present case the assumed duplication of the prochordal plate and anterior end of the notochord would presumably lead to duplication of the stomatodaeal region as a whole, and to two areas of persisting contact between neural and surface ectoderms, representing the anlagen of the two hypophyses. Mesoderm must then have surrounded and separated the two stomatodaeal areas from each 
other, forming mandibular and maxillary swellings around each primitive oral opening, and a common fronto-nasal swelling above them (Fig. 14). In this way normal structures would form laterally, and duplicates, to the limits of the available mesoderm, would form medially.

Each medial maxillary swelling would then have produced alveolar tissue containing a single central incisor tooth, and a lip element, which, by fusion with the lateral elements, formed complete alveolar and labial arches with a double frenulum marking the lines of fusion. A single fronto-nasal process would produce the normal external nose, and fuse with all four maxillary processes to complete the upper lip. Internally the lower margin of the nasal septum must carry remnants of the medial maxillary swellings to the upper part of the medial mandibular processes, represented by the abnormal bar of tissue. Ectoderm has persisted along this margin, forming squamous epithelium and hair follicles, and may have prevented closure of the palate (Scott, 1955). Partial development of mandibular structures on the medial side of each primitive oral opening accounts for the double lower lip, two symphyses menti and two tongues, the single tongue posteriorly forming from medial and lateral elements.

Additional adeno-hypophyseal gland tissue in the pharyngeal roof is regarded by many writers as being a variant of the normal (Patten, 1946), and Boyd (1956) drew attention to the constancy of such tissue in human embryos and adults. The extent of this tissue in the present case and its similarity with the main adeno-hypophysis indicate that it may have been capable of functioning as a source of internal secretions. Its presence on both sides of the median bar of tissue is in keeping with the course of development outlined above.

It is evident then that the abnormal structures in this case can be explained as being secondary to a duplication of the organizers for the primitive mouth region. No proper explanation for the absence of the olfactory tract can be offered, unless it be assumed that the derangement of growth in the brain and nasal region was sufficient to prevent development of the olfactory bulbs.

\section{Summary}

Duplication of the hypophysis and parts of the upper and lower jaws of a 38-week male infant are described.

Considerable masses of adeno-hypophyseal tissue were found in the roof of the mouth in positions corresponding to the stem regions of two Rathke's pouches.

An embryological explanation of the likely chain of events leading up to the conditions observed at birth is offered. This postulates that a transitory duplication of the anterior end of the inductor system for the head region occurred at about the fifteenth to sixteenth day of pregnancy, and led to the production of two primitive oral openings around which relatively normal development took place.

I am indebted to Dr. Muriel Frazer of the Jubilee Hospital, Belfast, for the opportunity of examining this case both before and after death, to Dr. H. T. Sheils for the family and clinical history, to Professor J. J. Pritchard for his interest in the preparation of the paper, and to Mr. George Bryans for assistance with the photography and the preparation of the large number of microscopic slides involved.

\section{REFERENCES}

Ahlfeld, F. (1880). Die Missbildungen des Menschen, pt. 1. Leipzig. Ancel, P. (1954). Arch. Anat. (Strasbourg), 37, pt. 2, p. 1.

Bosatra, A. (1955). Arch. ital. Otol., 66, 416.

Boyd, J. D. (1956). J. Anat. (Lond.), 90, 595.

Corner, G. W. (1955). Amer. J. Obstet. Gynec., 70, 933.

Fraser, F. C. and Fainstat, T. D. (1951). Amer. J. Dis. Child. $82,593$.

Gilbert, M. S. (1935). Anat. Rec., 62, 337.

Giroud, A. and Martinet, M. (1955). VIe Congrès Fédératif International d'Anatomie. Paris.

McCall, J. O., Jr. (1943). Anat. Rec., 87, 215

Patten, B. M. (1946). Human Embryology. Philadelphia.

Scott, J. H. (1955). J. Anat. (Lond.), 89, 561 .

Töndury, G. (1955). Münch. med. Wschr., 97, 1009. 